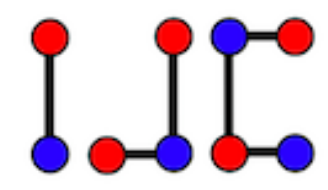

\title{
On additive vertex labelings
}

\author{
Christian Barrientos \\ Valencia College, Orlando, FL 32832, U.S.A. \\ chr_barrientos@yahoo.com
}

\begin{abstract}
In a quite general sense, additive vertex labelings are those functions that assign nonnegative integers to the vertices of a graph and the weight of each edge is obtained by adding the labels of its end-vertices. In this work we study one of these functions, called harmonious labeling. We calculate the number of non-isomorphic harmoniously labeled graphs with $n$ edges and at most $n$ vertices. We present harmonious labelings for some families of graphs that include certain unicyclic graphs obtained via the corona product. In addition, we prove that all $n$-cell snake polyiamonds are harmonious; this type of graph is obtained via edge amalgamation of $n$ copies of the cycle $C_{3}$ in such a way that each copy of this cycle shares at most two edges with other copies. Moreover, we use the edge-switching technique on the cycle $C_{4 t}$ to generate unicyclic graphs with another type of additive vertex labeling, called strongly felicitous, which has a solid bond with the harmonious labeling.
\end{abstract}

Keywords: additive vertex labelingt, harmonious, corona product, unicyclic, polyiamonds Mathematics Subject Classification: 05C30, 05C78

DOI: 10.19184/ijc.2020.4.1.5

\section{Introduction}

An additive vertex labeling of a graph $G$ of order $n$ and size $m$ is an injection from the vertex set of $G$ into a set $S$ (usually) of nonnegative integers, where each edge $u v$ of $G$ receives a weight defined as $f(u)+f(v)$. Several types of additive vertex-labelings have been studied over the last decades, harmonious labeling is among the most investigated. This labeling was introduced by Graham and Sloane in 1980 [16]. A graph $G$ of size $m$ is said to be harmonious if there

Received: 11 Jan 2020, Revised: 12 April 2020, Accepted: 20 April 2020. 
exists an injective function $f: V(G) \rightarrow \mathbb{Z}_{m}$ such that when each edge $u v$ of $G$ is assigned the weight $f(u)+f(v)$ reduced modulo $m$, the resulting weights are different, that is, they form the set $\mathbb{Z}_{m}=\{0,1, \ldots, m-1\}$. By extension, the function $f$ is also called harmonious. In Figure 1 we show an example of a harmoniously labeled graph together with its (extended) adjacency matrix. When $G$ is a tree, exactly one label may be used on two vertices. Graham and Sloane [16] conjectured that all trees are harmonious. In this direction, Figueroa-Centeno et al. [12] presented an interesting relationship between these labelings and the most restrictive difference vertex labeling, known as $\alpha$-labeling. They proved that any tree that admits an $\alpha$-labeling also admits a harmonious labeling.

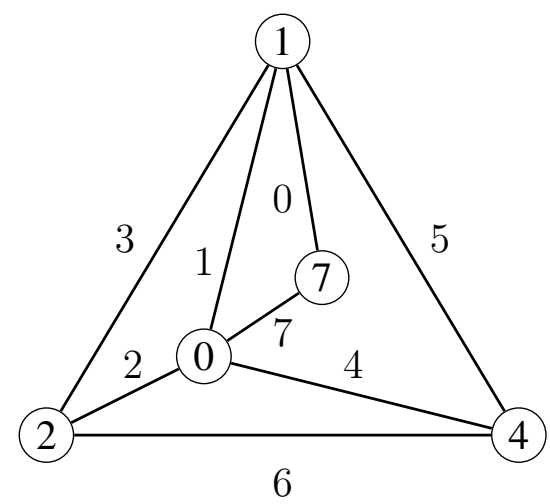

\begin{tabular}{l|l|l|l|l|l|l|l|l|}
+ & \multicolumn{1}{c}{0} & 1 & 2 & 3 & 4 & 5 & 6 & 7 \\
\cline { 2 - 9 } 0 & 0 & 1 & 2 & 3 & 4 & 5 & 6 & 7 \\
\cline { 2 - 9 } 1 & 1 & 2 & 3 & 4 & 5 & 6 & 7 & 0 \\
\cline { 2 - 9 } 2 & 2 & 3 & 4 & 5 & 6 & 7 & 0 & 1 \\
\cline { 2 - 9 } 3 & 3 & 4 & 5 & 6 & 7 & 0 & 1 & 2 \\
\cline { 2 - 9 } 4 & 4 & 5 & 6 & 7 & 0 & 1 & 2 & 3 \\
\hline 5 & 5 & 6 & 7 & 0 & 1 & 2 & 3 & 4 \\
\cline { 2 - 9 } 6 & 6 & 7 & 0 & 1 & 2 & 3 & 4 & 5 \\
\cline { 2 - 9 } 7 & 7 & 0 & 1 & 2 & 3 & 4 & 5 & 6 \\
\cline { 2 - 9 }
\end{tabular}

Figure 1. Harmonious labeling of a graph of order 5 and size 8, and its extended adjacency matrix

Theorem 1.1. If $T$ is an $\alpha$-tree, then $T$ is harmonious.

Since $\alpha$-labelings has been extensively studied, many families of graphs (trees) are known to accept an $\alpha$-labeling. Thus, Theorem 1.1 enlarges the number of known families of harmonious trees. Unfortunately, the converse of this theorem is false, there are trees that are harmonious but do not admit an $\alpha$-labeling, the smallest of these trees is the one obtained by subdividing every edge of the star $K_{1,3}$ exactly once. The reader interested in graph labelings is referred to [14] and [19].

Some of the general properties of harmonious graphs, proved by Graham and Sloane [16] are given below. In particular, theorems 1.3 and 1.4 are two methods to construct new harmonious graphs.

Theorem 1.2. If $f$ is a harmonious labeling of a graph of size $m$, then so is a $f+b$, where a is an invertible element of $\mathbb{Z}_{m}$ and $b$ is any element of $\mathbb{Z}_{m}$.

Corollary 1.1. Any vertex in a harmonious graph can be assigned the label 0 . Moreover, the repeated label in a harmonious tree can be any element of $\mathbb{Z}_{m}$. 
Theorem 1.3. Let $G$ be a harmoniously labeled graph containing (i) an edge uv of weight $w$, and (ii) a pair of vertices $x$, y not connected by an edge but satisfying $f(x)+f(y)=w$. Then deleting the edge uv and inserting xy changes $G$ to another harmonious graph.

Theorem 1.4. Let $T$ be a harmoniously labeled tree containing an edge uv of weight w, where $v$ is a leaf and $f(v)$ is the repeated label. If $x$ is any other vertex in $T$, we may delete the edge $u v$ and the vertex $v$ and replace them with a new vertex $y$ and edge $x y$ where $y$ is labeled with $f(y)=w-f(x)$.

In this work, we use these properties several times. Another result proved in [16] says that almost all graphs are not harmonious; in Section 2 we determine the number of harmoniously labeled graphs with as many vertices as edges. This number grows exponentially with the size of the graph.

We know the existence of at least three books whose major subject are additive vertex labelings, in particular those of the magic type (see [2], [19], and [25]). López and Muntaner-Batle [19] devoted in their work an entire chapter to harmonious labelings. Among the questions asked in [19] we have the following:

1. Are all lobsters harmonious?

2. Which unicyclic graphs are harmonious?

In Section 3, we use the corona product of two graphs to give partial answers to both questions. We prove that a harmonious lobster is obtained by attaching a pendant vertex to every vertex of a caterpillar if the difference of the cardinalities of the two stable sets of the caterpillar is at most one. Using caterpillars, we prove that any hairy cycle with odd girth is harmonious, that is, an odd cycle with pendant vertices attached to some of its vertices. As a corollary, we obtain a harmonious labeling of the corona between $C_{n}$ and $m K_{1}$ when $n$ is odd. We use a more restrictive kind of harmonious labeling to prove that if a graph $G$ admits this type of labeling, then so does its corona with $K_{1}$.

In [4], Barrientos and Minion proved that all snake polyominoes admit a harmonious labeling. Polyiamonds are a different version of Golomb's polyominoes. A $n$-cell snake polyiamond is a graph formed with $n$ copies of the cycle $C_{3}$ using edge amalgamation, in such a way that every triangle shares at most two edges. In Section 4 we present a harmonious labeling of these snakes. The labeling obtained satisfies the conditions to construct a harmonious labeling of the corona of any of these snakes and $K_{1}$.

In the last two sections we study additive vertex labelings, similar to the harmonious labelings, for the disjoint union of two harmonious graphs and for a family of unicyclic graphs obtained by switching edges of any cycle of size divisible by four. In Section 5 we extend the definition of harmonious labeling, given in [16], to include all graphs of order $n$ and size $n-1$. Trees fit in this category and as in the definition of harmonious labeling given by Graham and Sloane [16], we accept the repetition of exactly one label. With this extension, we prove that $G \cup K_{1, m}$ is harmonious provided that $G$ is sequential. In Section 6 we use the harmonious labeling of a path, given in [16], to construct a strongly felicitous labeling for any unicyclic graph obtained conveniently connecting $n \equiv 0(\bmod 4)$ copies of the path $P_{m}$. A strongly felicitous labeling is 
essentially the same that a harmonious labeling except that the largest label used is one unit larger than the size of the graph.

All graphs considered in this work are finite, with no loops nor multiple edges. All graph theoretical notation not given here is taken from [11] and [14].

\section{Enumerating Harmoniously Labeled Graphs}

All graphs considered in this section have size $n$ and order at most $n$. Suppose that $G$ is a harmonious graph of size $n$ and order at most $n$. Let $f$ be a harmonious labeling of $G$. The adjacency matrix of $G$ associated to this labeling can be embedded in the addition table of the additive group $\mathbb{Z}_{n}$. This embedding of the adjacency matrix of the graph in the addition table is what we call extended adjacency matrix. The facts that $G$ is a graph with no loops and its adjacency matrix is symmetric tell us that all the adjacencies of $G$ can be seen in the upper right triangle within the addition table. We can observe these properties in the addition table of $\mathbb{Z}_{8}$ given in Figure 1.

Since $\mathbb{Z}_{n}$ is an additive group, its addition table has exactly $n$ cells, $c_{i j}$, such that $i+j=n$. We want to determine the number of these cells that satisfy the additional condition $i<j$.

Suppose that $n$ is odd. Note that for each $k \in \mathbb{Z}_{n}$, there is one cell in the main diagonal, that is, a cell of the form $c_{i i}$, such that $i+i=k$. This implies that there are exactly $n-1$ cells $c_{i j}$, with $i \neq j$ where $i+j=k$. Therefore, $\frac{n-1}{2}$ of these cells satisfy the condition $i<j$.

Suppose that $n$ is even. In this case, the main diagonal only contains even numbers. Thus, if $k \in \mathbb{Z}_{n}$ is even, the cells $c_{i i}$, where $i=\frac{k}{2}$ and $i=\frac{n}{2}+\frac{k}{2}$, contain the integer $k$. Hence, for each even value of $k$, there are $n-2$ cells $c_{i j}$, with $i \neq j$, such that $i+j=k$; and only $\frac{n}{2}-1$ of them satisfy the condition $i<j$. When $k$ is odd, among the $n$ cells $c_{i j}$ containing the integer $k$, only $\frac{n}{2}$ satisfy $i<j$.

Now that we have determined the frequency of each $k \in \mathbb{Z}_{n}$ in the cells $c_{i j}$, with $i<j$, we may determine how many harmoniously labeled graphs of size $n$ and order at most $n$ exist.

Theorem 2.1. The number $a(n)$ of harmoniously labeled graphs of size $n$ and order at most $n$ is:

$$
a(n)= \begin{cases}\left(\frac{n-1}{2}\right)^{n} & \text { if } n \text { is odd, } \\ \left(\frac{n}{2}\right)^{\frac{n}{2}}\left(\frac{n}{2}-1\right)^{\frac{n}{2}} & \text { if n is even. }\end{cases}
$$

Proof. In order to induce the weights $0,1, \ldots, n-1$, any harmonious labeling of a graph of size $n$ and order at most $n$ assigns labels from $\mathbb{Z}_{n}$ to the vertices of the graph. This implies that inside the addition table of $\mathbb{Z}_{n}$, only one of the cells $c_{i j}$, with $i<j$, containing $k \in \mathbb{Z}_{n}$ is selected; if $c_{i j}$ is the cell selected, then the vertex labeled $i$ is connected to the vertex labeled $j$. In other terms, this triangular arrangement contains all the possible adjacencies; then, no matter what is the graph or its labeling, it is represented here. 
Suppose that $n$ is odd; every $k \in \mathbb{Z}_{n}$ appears $\frac{n-1}{2}$ times in this triangle, then there are $\left(\frac{n-1}{2}\right)^{n}$ possible ways to select $n$ different numbers from this triangle, each of the extended adjacency matrices obtained represents a different harmoniously labeled graph.

Similarly, when $n$ is even, every even $k \in \mathbb{Z}_{n}$ can be selected in $\frac{n}{2}-1$ different ways and every odd $k$ can be chosen in $\frac{n}{2}$ different forms. Thus, the number of different ways to select the $n$ cells in this triangle is given by $\left(\frac{n}{2}\right)^{\frac{n}{2}}\left(\frac{n}{2}-1\right)^{\frac{n}{2}}$, and this is the number of harmoniously labeled graphs of odd size $n$ and order at most $n$.

In the following table we show the initial values of $a(n)$. This is the sequence A329910 in the On-line Encyclopedia of Integer Sequences [6].

Table 1. Number of harmoniously labeled graphs of size $n$ and order at most $n$

\begin{tabular}{llllll}
\hline$n$ & $a(n)$ & $n$ & $a(n)$ & $n$ & $a(n)$ \\
\hline 1 & 0 & 2 & 0 & 3 & 1 \\
4 & 4 & 5 & 32 & 6 & 72 \\
7 & 2187 & 8 & 20736 & 9 & 262144 \\
10 & 3200000 & 11 & 48828125 & 12 & 729000000 \\
13 & 13060694016 & 14 & 230539333248 & 15 & 4747561509943 \\
16 & 96717311574016 & 17 & 2251799813685250 & 18 & 51998697814229000 \\
\hline
\end{tabular}

\section{Harmonious Graphs with Pendant Vertices}

Let $G$ and $H$ be graphs, the corona of $G$ and $H$, denoted by $G \odot H$, is the graph obtained by taking one copy of $G$ and as many copies of $H$ as vertices in $G$ and connecting, with an edge, the $i$ th vertex of $G$ to every vertex in the $i$ th copy of $H$. If $H \cong m K_{1}$, then $G \odot m K_{1}$ is a graph with pendant edges. A known family of harmonious graphs is the result of the corona of $C_{n}$ and $K_{1}$. Grace [15] showed that $C_{n} \odot m K_{1}$ is harmonious when $n$ is odd; Liu and Zhang [18] proved that $C_{n} \odot K_{1}$ is harmonious when $n$ is even. These graphs can be described as unicyclic graphs. A graph is said to be unicyclic if it has exactly one cycle, the length of this cycle is the girth of the graph; when it is connected, the graph has the same amount of vertices and edges and this implies that it contains at least three edges such that the deletion of any of them result in a tree. The interest in the study of graceful and harmonious labelings of unicyclic graphs can be traced to the works of Truszczyński [24] and Grace [15]. In [3], Barrientos proved that all cycles with pendant edges are graceful. Recently, Sethuraman and Murugan [23] presented new types of graceful unicyclic graphs. We are not aware of new results related to harmonious labelings of this kind of graphs.

In the following proposition, we study a family of unicyclic graphs that includes the coronas $C_{n} \odot m K_{1}$ when $n$ is odd. We prove that for any odd value of $n \geq 3$, the graph obtained attaching any number of pendant edges to all or some vertices of the cycle $C_{n}$ is a harmonious graph.

Firstly, some basic definitions. A leaf in a graph is a vertex of degree one. A caterpillar is a tree such that the removal of its leaves result in a path, which is called the spine of the caterpillar. A lobster is a tree such that the removal of its leaves produces a caterpillar, the spine of the underlying 
caterpillar is the spine of the lobster. A unicyclic graph $G$, other than a cycle, is called a hairy cycle if the deletion of any edge in the cycle results in a caterpillar. To label the vertices of $G$ we use the $\pi$-representation of bipartite graphs introduced by Rosa [21] and later used, among other authors, by Kotzig [17] and Cattell [9]. In this representation, the vertices of $G$ are arranged in two lines in such a way that each line contains all the vertices of one stable set of $V(G)$ and the edges connecting the vertices never cross. In Figure 2 we show the $\pi$-representation, providing a scheme of the harmonious labeling of caterpillars as given in [16]; Rosa [21] also used this representation for the $\alpha$-labeling of these trees.

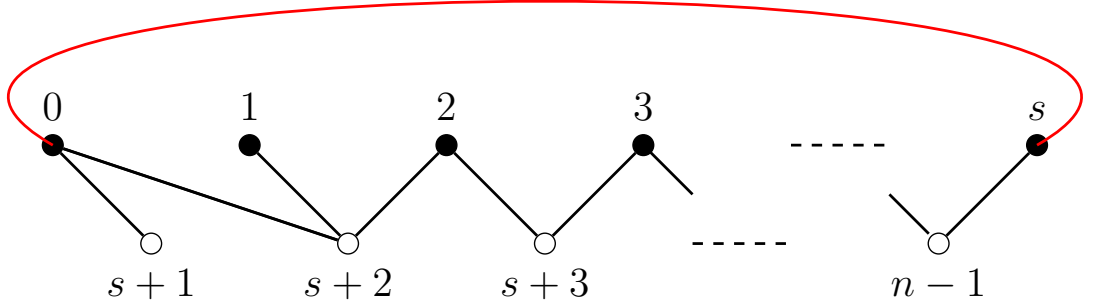

Figure 2. $\pi$-representation of a labeled caterpillar

Theorem 3.1. If $G$ is a hairy cycle with odd girth, then $G$ is harmonious.

Proof. Let $G$ be a hairy cycle of size $n$ with odd girth. There exists $u v \in E(G)$ such that $G^{\prime}=$ $G-e$ is a caterpillar. Since $G^{\prime}$ is bipartite, it can be drawn using the $\pi$-representation in such a way that $u$ and $v$ are located in the opposite extremes of one of the two lines of the arrangement; note that this happens because $G$ has odd girth. The labeling of $G^{\prime}$, shown in Figure 2, assigns the labels $0,1, \ldots, n-1$ to the vertices of $G^{\prime}$. The labels 0 and $s$ are assigned to $u$ and $v$, respectively.

Reading the diagram from left to right, the labels on each line are in ascending order; this implies that the edges of $G^{\prime}$ have weights $s+1, s+2, \ldots, s+n-1$. If we reconnect the vertices $u$ and $v$ (the red edge in the diagram) the edge $e=u v$ has weight $s$. In other terms, the labeling of $G$ induces $n$ different weights, which are consecutive integers. Therefore, if these integers are reduced modulo $n$, we obtain the elements of $\mathbb{Z}_{n}$ and the labeling of $G$ is harmonious.

In Figure 3 we show an example of this labeling where $G$ has size 20, girth 11, and $s=6$, the edge $e=u v$ is represented in red.

As a consequence of this theorem we get Grace's result about the harmonious labeling of $C_{n} \odot m K_{1}[15]$.

Corollary 3.1. For every $m \geq 1$ and $n \geq 3$ odd, the corona $C_{n} \odot m K_{1}$ is a harmonious graph.

We say that a tree is neutral if the difference of the cardinalities of its stable sets is at most one. In the following result we prove that a harmonious neutral caterpillar can be extended to a harmonious neutral lobster. In order to prove our claim, we use the labeling scheme of a caterpillar exhibited in Figure 2. 


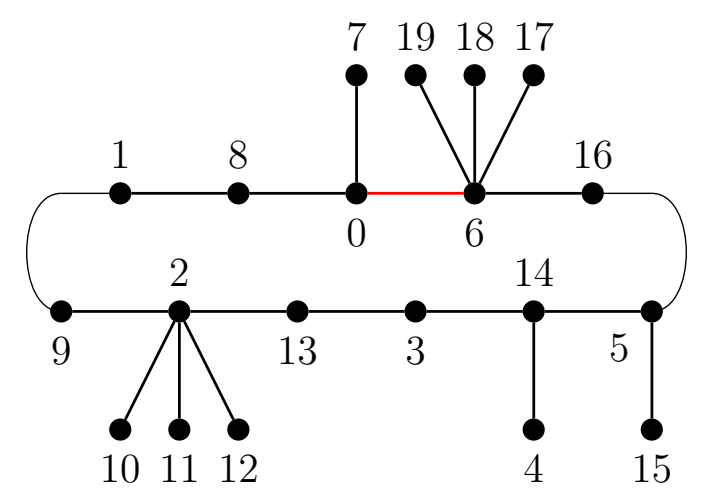

Figure 3. Harmonious labeling of a unicyclic graph

Theorem 3.2. Let $G$ be a caterpillar of size $n$. If $G$ is neutral, then $G \odot K_{1}$ is harmonious.

Proof. Suppose that $G$ is a neutral caterpillar of size $n$ with stable sets $A$ and $B$ such that $a=$ $|A| \geq|B|=b$. Before labeling $G$, we draw the graph using the $\pi$-representation given in Figure 2. Starting on the left side, the elements of $A$ are assigned the labels $0,1, \ldots, a-1$, in a sequential manner; similarly, the vertices of $B$ receive the labels $a, a+1, \ldots, n$. In this way, the weights induced on the edges of $M$ are $a, a+1, \ldots, a+n-1$.

The assignment of labels to the $n+1$ pendant vertices depends on whether $a$ equals $b$ or not. Suppose that $a=b$. The pendant vertices adjacent to the elements of $B$ are labeled with the integers $n+1, n+2, \ldots, n+b$; those adjacent to the elements of $A$ are labeled with the integers $n+b, n+b+1, \ldots, n+2 b-1$. Since $a=b$ and $a+b-1=n$, the last (or largest) label assigned to one of these vertices is $2 n$. Thus, the pendant edges incident to the vertices in $A$ have weights $a+n, a+n+2, \ldots, 2 n+a-1$; the others pendant edges have weights $a+n+1, a+n+3, \ldots, 2 n+a$.

Suppose now that $a=b+1$. In this case, the pendant vertices adjacent to the vertices of $B$ are labeled with the integers $n+1, n+2, \ldots, n+b$, while the other pendant vertices are labeled $n+a, n+a+1, \ldots, 2 n+1$. The weights of the pendant edges incident to the elements of $A$ are $n+a, n+a+2, \ldots, 2 n+a$. On the other side, the pendant edges have weights $n+a+1, n+$ $a+3, \ldots 2 n+b=2 n+a-1$.

Hence, the labels assigned to the vertices of $G \odot K_{1}$ are $0,1, \ldots, 2 n+1$ (where the label $2 n+1$ must be reduced modulo $2 n+1$ ) and the induced weights are $a, a+1, \ldots, 2 n+1$. When the label $2 n+1$ is reduced modulo $2 n+1$, the edge of weight $2 n+a$ has weight $a-1$; i.e., the weights are $2 n+1$ consecutive integers and the labeling of the corona between the neutral caterpillar $G$ and $K_{1}$ is harmonious.

We show a couple of examples in Figure 4, the first one for the case where $a=b$ and the second one for the case where $a>b$.

Since the maximum distance from any vertex of $G \odot K_{1}$ to the spine of $G$ is two, the tree $G \odot K_{1}$ is in fact a lobster. Thus, Theorem 3.1 partially answers the question posed in the Introduction 

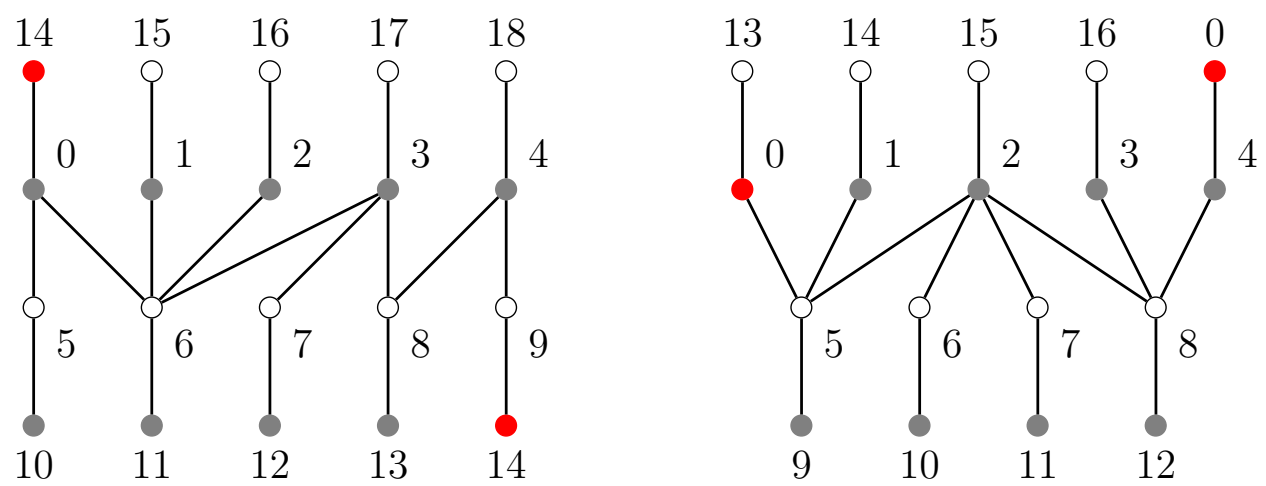

Figure 4. Harmonious labeling of $G \odot K_{1}$, where $G$ is a neutral caterpillar

about the harmoniousness of lobsters. Taking under consideration the characteristics of this lobster, the last theorem could be written in the following terms.

Theorem 3.3. If $L$ is a neutral lobster of odd size such that every vertex is a leaf or adjacent to exactly one leaf, then $L$ is harmonious.

The upcoming propositions involve the two specific results that can be found in [16]. There, Graham and Sloane showed the harmonious labeling for two graphs constructed using graph operations. In particular, they proved that the fan $F_{n}=P_{n}+K_{1}$ and the ladder $L_{n}=P_{n} \times K_{2}$ are harmonious graphs. In the following propositions we prove that when $n$ is odd, both $F_{n} \odot K_{1}$ and $L_{n} \odot K_{1}$ are harmonious graphs. The harmonious labeling of $F_{n}$ and $L_{n}$ used in the proofs of these propositions are the labelings given by Graham and Sloane [16], so we do not prove here that fact.

Recall that for $n \geq 2$, the fan $F_{n}$ is obtained by joining all the vertices of the path $P_{n}$ to a new vertex, called the center; that is, $F_{n}=P_{n}+K_{1}$. For $n=2 t+1$, the fan $F_{n}$ is a graph of order $2 t+2$ and size $4 t+1$.

Proposition 3.1. For every odd value of $n \geq 1$, the corona $F_{n} \odot K_{1}$ is harmonious.

Proof. When $n=1$, the fan $F_{1}$ is the path $P_{2}$ which implies that it is harmonious. The harmonious labeling of $F_{3}$ is shown in Figure 5 inside the labeling of $F_{3} \odot K_{1}$. Let $t \geq 2$ be an integer such that $n=2 t+1$. Suppose that $f$ is the harmonious labeling of $F_{2 t+1}$ given in [16]. That is, if $v_{1}, v_{2}, \ldots, v_{2 t+1}$ are the consecutive vertices of $P_{2 t+1}$ and $v_{0}$ is the center of $F_{2 t+1}$, then

$$
f\left(v_{i}\right)= \begin{cases}0 & \text { if } i=0, \\ t+\frac{i-1}{2} & \text { if } i \text { is odd } \\ 2 t+\frac{i}{2} & \text { if } i \text { is even. }\end{cases}
$$

For each $i \in\{0,1, \ldots, 2 t+1\}$, let $u_{i}$ be the pendant vertex adjacent to $v_{i}$. We extend the labeling $f$ to include these vertices, according to the following rule: 


$$
f\left(u_{i}\right)= \begin{cases}t-1 & \text { if } i=0 \\ 4 t+\frac{i+1}{2} & \text { if } i \text { is odd } \\ 3 t+\frac{i}{2} & \text { if } i>0 \text { is even. }\end{cases}
$$

Recall that when $f$ is restricted to $F_{2 t+1}$, it is harmonious, which implies that if the induced weights are not reduced modulo $4 t+1$, they are $t, t+1, \ldots, 5 t$. The edge $u_{0} v_{0}$ has weight $t-1$ and for each $i \in\{1,2, \ldots, m\}$, the edge $u_{i} v_{i}$ has weight $5 t+i$; in other terms, $5 t+1,5 t+2, \ldots, 7 t+1$. Then, the set of weights induced by $f$ on the edges of $F_{2 t+1} \odot K_{1}$ is $\{t-1, t, \ldots, 7 t+1\}$; i.e., a set with $6 t+3=3(2 t+1)=3 n$ elements. Since the assignment of labels is injective and all the labels are in the range $\{0,1, \ldots, 5 t+1\}$ and $5 t+1$ is smaller that the size $6 t+3$ of $F_{2 t+1} \odot K_{1}$, we conclude that $f$ is a harmonious labeling of this graph.

In Figure 5 we show the harmonious labelings of $F_{3} \odot K_{1}$ and $F_{7} \odot K_{1}$ obtained using the labeling given in the proof of the above proposition. The harmonious labeling of $F_{3}$ was taken directly from [16].
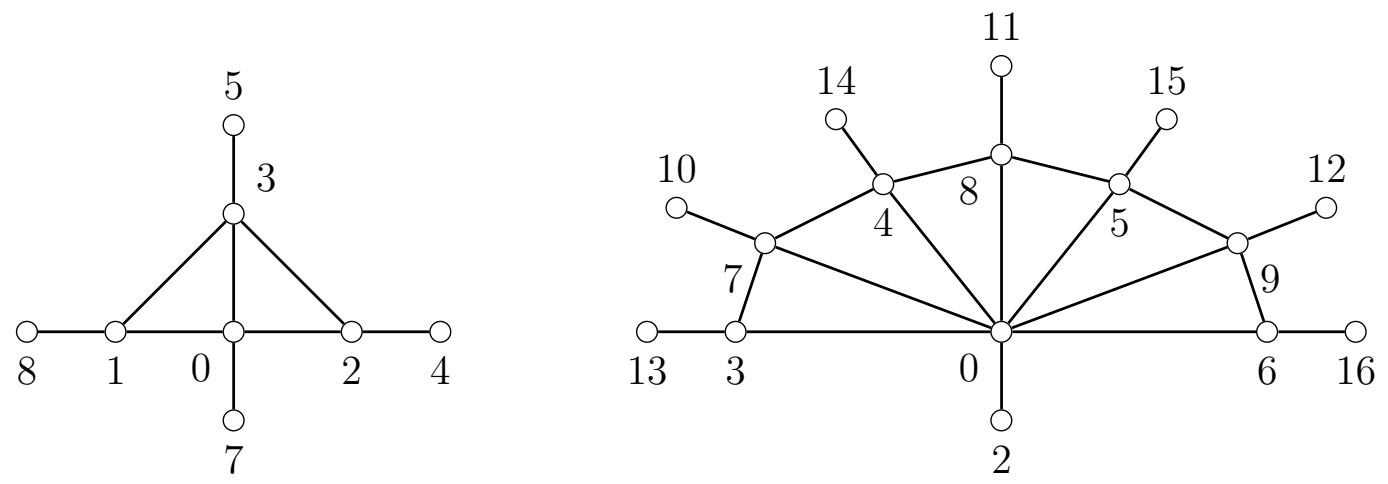

Figure 5. Harmonious labeling of $F_{3} \odot K_{1}$ and $F_{7} \odot K_{1}$

In the next proposition we prove that the corona $L_{2 t+1} \odot K_{1}$ is harmonious. This graph has order $8 t+4$ and size $10 t+3$.

Proposition 3.2. The corona $L_{2 t+1} \odot K_{1}$ is harmonious.

Proof. Suppose that $V\left(L_{2 t+1}\right)=\left\{v_{i}: 1 \leq i \leq 4 t+2\right\}$ and $E\left(L_{2 t+1}\right)=\left\{v_{i} v_{i+1}: 1 \leq i \leq\right.$ $2 t\} \cup\left\{v_{i} v_{i+1}: 2 t+2 \leq i \leq 4 t+1\right\} \cup\left\{v_{i} v_{2 t+1+i}: 1 \leq i \leq 2 t+1\right\}$. We use the harmonious labeling of the vertices of $L_{2 t+1}$ given in [16]. Thus, 


$$
f\left(v_{i}\right)= \begin{cases}\frac{i-1}{2} & \text { if } 1 \leq i \leq 2 t+1 \text { is odd, } \\ t+\frac{i}{2} & \text { if } 2 \leq i \leq 2 t \text { is even, } \\ t+\frac{i-1}{2} & \text { if } 2 t+3 \leq i \leq 4 t+1 \text { is odd } \\ 2 t+\frac{i}{2} & \text { if } 2 t+2 \leq i \leq 4 t+2 \text { is even. }\end{cases}
$$

Therefore, the vertices of the ladder are labeled with the integers $0,1, \ldots, 4 t+1$; the induced weights are $t+1, t+2, \ldots, 7 t+1$.

For each $i \in\{0,1, \ldots, 4 t+1\}$, we denote by $u_{i}$ the pendant vertex attached to the vertex of $L_{2 t+1}$ labeled $i$. We extend $f$ to the vertices $u_{i}$ in the following form:

$$
f\left(u_{i}\right)= \begin{cases}7 t+3+i & \text { if } 0 \leq i \leq 2 t \\ 3 t+i & \text { if } 2 t+1 \leq i \leq 4 t+1\end{cases}
$$

Clearly, this extension of $f$ is injective and its range is $\{5 t+1,5 t+2, \ldots, 9 t+3\}-\{7 t+2\}$. Furthermore, for each $i \in\{0,1, \ldots, 2 t\}$, the edge incident to $u_{i}$ has weight $7 t+3+2 i$. So, these pendant edges have weights $7 t+3,7 t+5, \ldots, 11 t+3$. For each $i \in\{2 t+1,2 t+2, \ldots, 4 t+1\}$, the edge incident to $u_{i}$ has weight $3 t+2 i$. In this case, the weights obtained are $7 t+2,7 t+4, \ldots, 11 t+2$.

Hence, the labels assigned by $f$ on the vertices of $L_{2 t+1} \odot K_{1}$ are in the range $\{0,1, \ldots, 9 t+3\}$ and the induced weights are $t+1, t+2, \ldots, 11 t+3$. Therefore, $f$ is a harmonious labeling of the corona $L_{2 t+1} \odot K_{1}$.

In the Figure 6 we show these labeled graphs for the cases $t=1,2,3$.
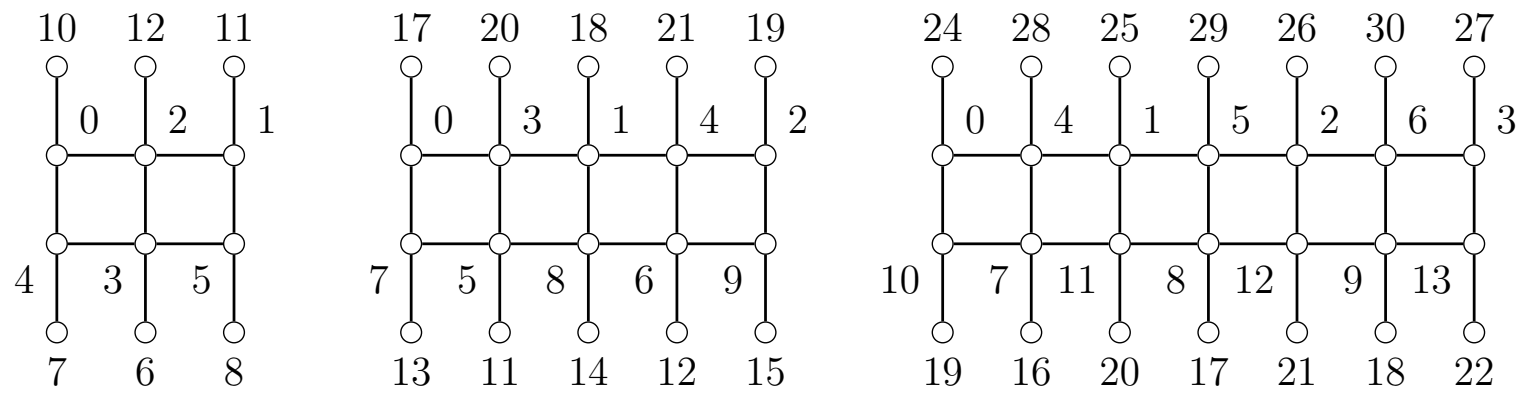

Figure 6. Harmonious labeling of the corona $L_{2 t+1} \odot K_{1}$ 


\section{The $n$-Cell Snake Polyiamonds}

Polyiamonds are geometrical shapes constructed from unit equilateral triangles (or the cycle $C_{3}$ from a graph theoretical perspective) joined edge-to-edge on a triangular grid. Here, we are interested into $n$-cell snake polyiamonds, i.e., those shapes where each, of $n$ triangles, shares at most two edges with other triangles.

Related to snake polyiamonds is the concept of rigid ladder introduced by the Agnarsson and Greenlaw [1]. Here, we extend their definition. We say that a graph $G$ of order $2 n$ is a (generalized) rigid ladder if its vertex set is $\left\{u_{i}, v_{i}: 1 \leq i \leq n\right\}$ and its edges are, for every $1 \leq i \leq n$, of the form $u_{i} v_{i}$, and for every $1 \leq i \leq n-1$, of the forms $u_{i} u_{i+1}, u_{i+1} v_{i}$, and either $u_{i} v_{i+1}$ or $u_{i+1} v_{i}$, but not both. In other terms, $G$ is obtained from the ladder $L_{n}$ by adding for each square a "diagonal", either ascending or descending. In [1] all the diagonals are parallel. This generalized version of the original definition was given in [8]. There, we defined what is a rigid grid in exactly the same way that we did with the ladders. That is, a rigid grid is the graph obtained from $P_{m} \times P_{n}$ by adding a diagonal for each square. We calculated the number of non-isomorphic rigid grids for every $m \geq 2$ and $n \geq 2$. The number of non-isomorphic rigid ladders of order $2 n+2$, that is, those formed from $P_{n+1} \times P_{2}$, is

$$
a(n)=2^{n-2}+2^{\left\lfloor\frac{n}{2}\right\rfloor-1}
$$

The sequence A005418 in OEIS [6] is formed by the consecutive values of $a(n)$. All the rigid ladders of order $2 n+2$ correspond to $n$-cell snake polyiamonds; however, there are snakes that are not rigid ladders, for instance, the snakes on the second row of Figure 7. Then, the number $a(n)$ can be understood as a lower bound for the number of non-isomorphic $n$-cell snake polyiamonds.

Suppose that $G$ is a $n$-cell snake polyiamond. Thus, $G$ is an outerplanar graph of order $n+2$ and size $2 n+1$ whose dual graph is the fan $F_{n}$. The vertices of $G$ can be colored with two colors, say red and black, in such a way there are no monochromatic cells in $G$. Consequently, the vertices of $G$ can be organized in two monochromatic paths: $P_{\left\lfloor\frac{n+2}{2}\right\rfloor}$ containing the red vertices, and $P_{\left\lceil\frac{n+2}{2}\right\rceil}$ containing the black vertices. If the consecutive black vertices are $v_{1}, v_{2}, \ldots, v_{\left\lceil\frac{n+2}{2}\right\rceil}$ and the consecutive red vertices are $u_{1}, u_{2}, \ldots, u_{\left\lfloor\frac{n+2}{2}\right\rfloor}$, we assume that $u_{1} v_{1}$ and $u_{\left\lfloor\frac{n+2}{2}\right\rfloor} v_{\left\lceil\frac{n+2}{2}\right\rceil}$ are edges of $G$. In the next theorem we prove that all $n$-cell snake polyiamonds are harmonious.

Theorem 4.1. For every $n \geq 1$, the $n$-cell snake polyiamond is a harmonious graph.

Proof. Suppose that $G$ is a $n$-cell snake polyiamond. Let $f$ be a labeling of the vertices of $G$ defined as $f\left(v_{i}\right)=2 i-2$ for each $1 \leq i \leq\left\lceil\frac{n+2}{2}\right\rceil$ and $f\left(u_{j}\right)=2 j-1$ for each $1 \leq j \leq\left\lfloor\frac{n+2}{2}\right\rfloor$. Clearly, $f$ is an injective function which range is $\{0,1, \ldots, n+1\}$. Note that the label $n+1$ is located on a red vertex when $n$ is odd and on a black vertex when $n$ is even.

Since the black vertices are labeled with consecutive even numbers, the weights induced by $f$ on the edges of $P_{\left\lceil\frac{n+2}{2}\right\rceil}$ are consecutive integers congruent to $2(\bmod 4)$. On the other side, the red vertices are labeled with consecutive odd numbers, so the weights of the edges of $P_{\left\lfloor\frac{n+2}{2}\right\rfloor}$ are consecutive integers congruent to $0(\bmod 4)$. The remaining edges of $G$ have end-vertices of different colors, which implies that their weights are odd numbers. Regardless the parity of $n$, the edge $u_{1} v_{1}$ has weight 1 and the weight of $u_{\left\lfloor\frac{n+2}{2}\right\rfloor} v_{\left\lceil\frac{n+2}{2}\right\rceil}$ is $2 n+1$. 
Suppose that $u_{p} v_{q}$ and $u_{r} v_{s}$ are two of these edges. Their weights are:

$$
f\left(u_{p}\right)+f\left(v_{q}\right)=(2 p-1)+(2 q-2)=2(p+q)-3
$$

and

$$
f\left(u_{r}\right)+f\left(v_{s}\right)=(2 r-1)+(2 s-2)=2(r+s)-3 .
$$

Since $G$ is an outerplanar graph and the two edges are different, then one of the following holds: (1) $p<r$ and $q<s$, (2) $p=r$ and $q<s$, or (3) $p<r$ and $q=s$. Each of these conditions implies that $p+q<r+s$. Consequently, the weight of $u_{p} v_{q}$ is different of the weight of $u_{r} v_{s}$. Therefore, the weights of the edges with end-vertices of different colors are all the odd numbers from 1 up to $2 n+1$. In addition, the weights of the edges with end-vertices of the same color are all the even numbers from 2 up to $2 n+2$; that is, $0,2, \ldots, 2 n(\bmod 2 n+2)$.

Hence, $f$ is a harmonious labeling of $G$.

In Figure 7 we show all the 6-cell snake polyiamonds labeled using the function described in the proof.
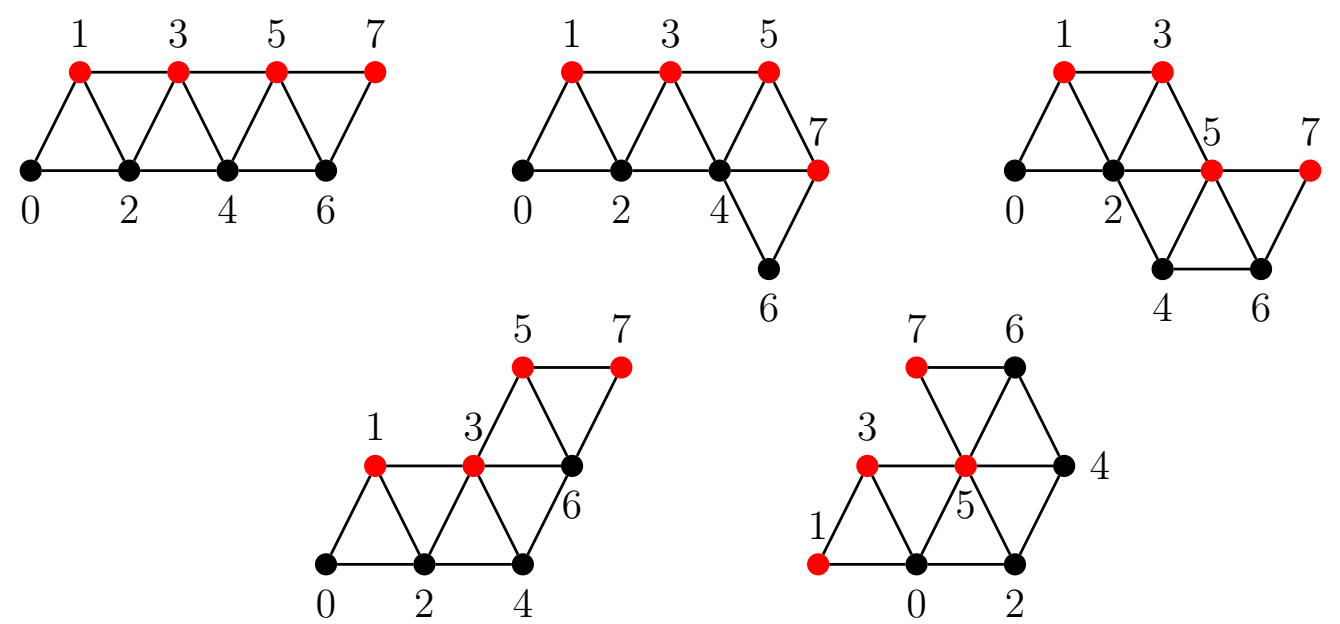

Figure 7. Harmonious labelings of all 6-cell snake polyiamonds

Note that this labeling of the $n$-cell snake polyiamonds $G$ assigns labels that are consecutive integers, the same that the labelings of the fan $F_{n}$ and the ladder $L_{n}$ given before. Hence, using the same type of argument it can be proved that the corona of $G$ and $K_{1}$ is a harmonious graph. We omit the proof of this claim.

Proposition 4.1. If $G$ is a n-cell snake polyiamond, then the corona $G \odot K_{1}$ is harmonious.

\section{Harmonious Labelings of Disconnected Graphs}

In this section we present two results about harmonious labelings of disconnected graphs. There are several results about this type of graph: Youssef [27] proved that the disjoint union 
of an odd number of copies of a harmonious graph is a harmonious graph. Seoud et al. [22] proved that $C_{n} \cup C_{n+1}$ is harmonious if and only if $n \geq 4$. Yang et al. [26] showed that all $C_{2 n+1} \cup C_{2 m}$ are harmonious except for $C_{3} \cup C_{4}$. Renuka et al. [20] showed that $C_{2 t+1} \cup P_{3}$ and $C_{2 t+1} \odot m K_{1} \cup P_{3}$ are harmonious. For more detailed information about harmonious labelings of disconnected graphs, we refer the reader to Gallian's survey [14].

Recall that in a harmonious labeling of a tree of size $n$, the labels are taken from $\mathbb{Z}_{n}$ and exactly one of them must be used twice. Trees are members of a larger family of graphs, the members of this family are all the graphs of size $n$ and order $n+1$, we denote this family by $\mathscr{D}$. We extend the definition of harmonious labeling to include the elements of $\mathscr{D}$, not only trees, that is, if $G$ is any harmonious graph of size $n$ and order $n+1$, then its vertices can be labeled with integers from $\mathbb{Z}_{n}$ and exactly one of the labels can be used twice. Among the elements of $\mathscr{D}$ we have all disconnected graphs formed by the union of a unicyclic graph and a tree, we also have in $\mathscr{D}$ the union of a 2-regular graph and a tree.

Grace [15] defined a sequential labeling of a graph of size $q$ as an additive vertex-labeling with range $\mathbb{Z}_{q}$, where the set of induced weights consists of $q$ consecutive integers. A graph that admits a sequential labeling is called sequential. In the following theorem we prove that if $G \in \mathscr{D}$ is sequential, then the union of $G$ and the star $K_{1, m}$ is a harmonious graph.

Theorem 5.1. Let $G$ be a graph of order $n$ and size $n$. If $G$ is sequential, then $G \cup K_{1, m}$ is harmonious.

Proof. Note that the graph $G \cup K_{1, m}$ has order $n+m+1$ and size $n+m$. This implies that any harmonious labeling of this graph must repeat one label. Let $f$ be a sequential labeling of $G$ and $\omega$ be the maximum weight induced by $f$. The vertex of degree $m$ of $K_{1, m}$ is labeled $\omega+1-n$ and the vertices of degree 1 are labeled with the integers $n, n+1, \ldots, n+m-1$. In this way, the edges of $K_{1, m}$ have weights $\omega+1, \omega+2, \ldots, \omega+m$.

Therefore, the weights on the edges of $G \cup K_{1, m}$ are $n+m$ consecutive integers, that when reduced $(\bmod n+m)$ they are the elements of $\mathbb{Z}_{n+m}$. Since the labels on $G$ are $0,1, \ldots, n-1$ and the labels on the vertices of $K_{1, m}$ are $n, n+1, \ldots, n+m-1$ and $\omega+1-n$, every label is used exactly once except $\omega+1-n$ that is used on $G$ and $K_{1, m}$ because $\omega$ cannot exceed the value $2 n-3$. Consequently, the labeling of $G \cup K_{1, m}$ is harmonious.

Recall that in Theorem 3.1 we proved that all hairy cycles are harmonious. These graphs satisfy the conditions of the previous theorem, therefore, its union with any star results in a harmonious graph.

Corollary 5.1. If $G$ is a hairy cycle, then $G \cup K_{1, m}$ is a harmonious graph.

In Figure 8 we show an example of the labeling in Theorem 5.1 for the graph $C_{9} \cup K_{1,7}$.

\section{Strongly Felicitous Unicyclic Graphs}

Suppose that $n \equiv 0(\bmod 4)$. Let $P^{1}, P^{2}, \ldots, P^{n}$ be copies of the path $P_{m}$, with vertex set $V\left(P^{i}\right)=\left\{u_{i, j}: 1 \leq j \leq m\right\}$ and edge set $E\left(P^{i}\right)=\left\{u_{i, j} u_{i, j+1}: 1 \leq j<m\right\}$. Using these paths we want to generate a unicyclic graph of order $n m$; to do so, for every $i \in\{1,2, \ldots, n-1\}$ we 

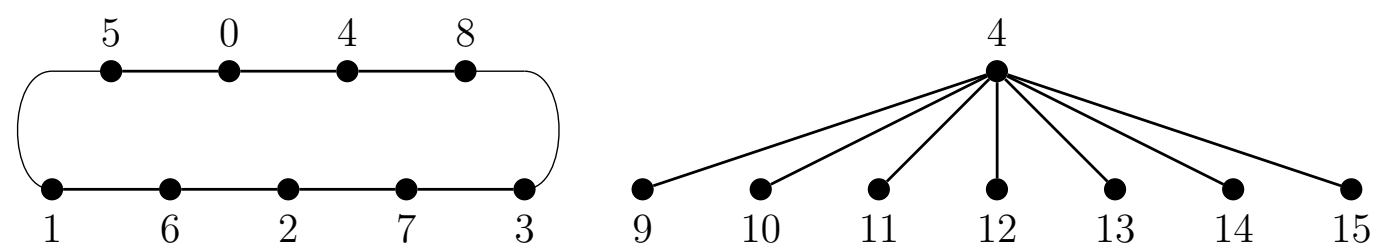

Figure 8. Harmonious labeling of $C_{9} \cup K_{1,7}$

select an index $k \in\{1,2, \ldots, m\}$ and connect the vertices $u_{i, k}$ and $u_{i+1, k}$. When this process has been done for every $i \in\{1,2, \ldots, n-1\}$, the resulting graph is a tree because it is connected and its size is $n(m-1)+n-1=n m-1$. Finally, in order to create a unicyclic graph a new edge must be introduced. In our process, we have two ways to select the pair of vertices to be connected. Assume that $u_{\frac{n}{2}, k} u_{\frac{n}{2}+1, k}$ is an edge of this tree.

1. If $k$ is even, then select any odd number $j \in\{1,2, \ldots, m\}$ other than $k$ and connect $u_{\frac{n}{2}, j}$ with $u_{\frac{n}{2}+1, j}$. Since $j \neq k, u_{\frac{n}{2}, j} u_{\frac{n}{2}+1, j}$ is a new edge and the resulting graph is unicyclic.

2. If $k$ is odd, then we have two alternatives to build a unicyclic graph:

(a) Select any even number $j \in\{1,2, \ldots, m\}$ other than $k$ and proceed as in the previous case.

(b) Connect the vertices $u_{1,1}$ and $u_{n, m}$. Since $P^{1}$ and $P^{n}$ were not connected before, the new graph is unicyclic.

We denote by $\mathscr{F}$ the family of all unicyclic graphs of order $n m$ obtained using the construction described above. We claim that any graph in $\mathscr{F}$ admits another type of additive vertex labeling, named strongly felicitous by Figueroa-Centeno et al. [13].

An injective function $f$ from the vertices of a graph $G$ with $m$ edges to the set $\{0,1, \ldots, m\}$ is called felicitous if the weights induced by $f(u)+f(v)(\bmod m)$ for each edge $u v$ are distinct. If there exists and integer $\lambda$ such that $\min \{f(u), f(v)\} \leq \lambda<\max \{f(u), f(v)\}$, then $f$ is called strongly felicitous. In [13], the authors proved that a graph of order $n$ and size $m$, with $m \geq n-1$, is strongly felicitous if and only if it is an $\alpha$-graph.

Every unicyclic graph satisfies this condition between order and size; therefore, proving that it is an $\alpha$-graph or strongly felicitous is equivalent. In [9], we proved the existence of an $\alpha$-labeling for a family of graphs that includes all unicyclic graphs where two vertices of $P_{\frac{n}{2}}$ are connected with two vertices of $P_{\frac{n}{2}}+1$. In the following proposition we prove that all members of $\mathscr{F}$ are strongly felicitous.

In order to facilitate the description of the unyclic graph and its labeling, we rename its vertices according to the following formula:

$$
\phi\left(u_{i, j}\right)= \begin{cases}v_{1, m(i-1)+j} & \text { if } i<\frac{n}{2} \text { is odd, } \\ v_{1, m i+1-j} & \text { if } i \leq \frac{n}{2} \text { is even, } \\ v_{1, m(i-1)+j} & \text { if } i>\frac{n}{2} \text { is odd, } \\ v_{1, m i+1-j} & \text { if } i>\frac{n}{2} \text { is even. }\end{cases}
$$


Hence, independently of the parity of $i$ or how it compares with $\frac{n}{2}$, the values of $m(i-1)+j$ and $m i+1-j$, for all $j \in\{1,2, \ldots, m\}$, form a set of $2 m$ consecutive integers. Therefore, $\phi$ is a bijection. We use this bijection to define, in a more efficient way, the labeling of all the members of $\mathscr{F}$.

Proposition 6.1. If $G \in \mathscr{F}$, then $G$ is strongly felicitous.

Proof. To build our strongly felicitous unicyclic graph $G$, we start with two disjoint copies of $P_{\frac{n m}{2}}$. For each $i \in\{1,2\}$, let $R_{i}$ be the $i$ th copy of $P_{\frac{n m}{2}}$, where $v_{i, 1}, v_{i, 2}, \ldots, v_{i, m}$ are its consecutive vertices. We define a labeling $f$ of $R_{1} \cup R_{2}$ as follows:

$$
f\left(v_{i, j}\right)= \begin{cases}\frac{j-1}{2} & \text { if } i=1 \text { and } j \text { is odd, } \\ \frac{n m}{2}+\frac{j}{2} & \text { if } i=1 \text { and } j \text { is even, } \\ \frac{n m}{4}+\frac{j+1}{2} & \text { if } i=2 \text { and } j \text { is odd, } \\ \frac{3 n m}{4}+\frac{j}{2} & \text { if } i=2 \text { and } j \text { is even. }\end{cases}
$$

It is not hard to see that the labels on $R_{1}$ and $R_{2}$ are $\left\{0,1, \ldots, \frac{n m}{4}-1\right\} \cup\left\{\frac{n m}{2}+1, \frac{n m}{2}+\right.$ $\left.2, \ldots, \frac{3 n m}{4}\right\}$ and $\left\{\frac{n m}{4}+1, \frac{n m}{4}+2, \ldots, \frac{n m}{2}\right\} \cup\left\{\frac{3 n m}{4}+1, \frac{3 n m}{4}+2, \ldots, n m\right\}$, respectively.

Suppose that $i=1$. Independently of the parity of $j$, the weight of $v_{1, j} v_{1, j+1}$ is $\frac{n m}{2}+j$. This implies that the weights induced on the edges of $R_{1}$ are $\frac{n m}{2}+1, \frac{n m}{2}+2, \ldots, n m$.

Suppose that $i=2$. Similarly to the case $i=1$, the weight of $v_{2, j} v_{2, j+1}$ is $\frac{n m}{2}+j+1$ no matter the parity of $j$. Hence, the weights induced on the edges of $R_{2}$ are $n m+2, n m+3, \ldots, \frac{3 n m}{2}$.

When $v_{1, \frac{n m}{2}}$ is connected to $v_{2,1}$, we obtain an edge of weight $\frac{3 n m}{4}=\frac{n m}{4}+1=n m+1$. When $v_{1,1}$ is connected to $v_{2}, \frac{n m}{2}$, we get an edge of weight $0+\frac{3 n m}{4}+\frac{n m}{4}=n m$. Thus, the graph acquired, when these edges are added, is the cycle $C_{n m}$, which is a member of $\mathscr{F}$. The labels used are in the range $\{0,1, \ldots, n m\}-\left\{\frac{n m}{4}\right\}$ and the induced weights are $\frac{n m}{2}+1, \frac{n m}{2}+2, \ldots, \frac{3 n m}{2}$.

Now we use this labeled cycle to get our unicyclic graph $G$. Let $0<k<\frac{n m}{2}$ be any multiple of $m$ and $s \in\{1,2, \ldots, m-1\}$. Note that the edge $v_{1, k} v_{1, k+1}$ has weight $\frac{n m}{2}+k$ and that

$$
f\left(v_{1, k-s}\right)+f\left(v_{1, k+1+s}\right)=\frac{k-s-1}{2}+\frac{n m}{2}+\frac{k+1+s}{2}=\frac{n m}{2}+k .
$$

This implies that the edge $v_{1, k} v_{1, k+1}$ can be replaced by the new edge $v_{1, k-s} v_{1, k+1+s}$ and the new graph has the same weights that $C_{n m}$. Similarly, on $R_{2}$, the edge $v_{2, k} v_{2, k+1}$ has weight $n m+k+1$ and

$$
f\left(v_{2, k-s}\right)+f\left(v_{2, k+1+s}\right)=\frac{n m}{4}+\frac{k-s+1}{2}+\frac{3 n m}{2}=n m+k+1
$$

Consequently, the edge $v_{2, k} v_{2, k+1}$ can be substituted by $v_{2, k-s} v_{2, k+1+s}$ without affecting the set of induced weights. 
With the edges $v_{1, \frac{n m}{2}} v_{2,1}$ and $v_{1,1} v_{2, \frac{n m}{2}}$ we proceed in a slightly different way. First we must observe that these edges have weights $n m+1$ and $n m$, respectively. For every even value of $s \in\{1,2, \ldots, m\}$, the edge $v_{1, \frac{n m}{2}} v_{2,1}$ can be replaced with $v_{1, \frac{n m}{2}-s} v_{2,1+s}$ because

$$
\begin{aligned}
f\left(v_{1, \frac{n m}{2}-s}\right)+f\left(v_{2,1+s}\right) & =\left(\frac{n m}{2}+\frac{1}{2}\left(\frac{n m}{2}-s\right)\right)+\left(\frac{n m}{4}+\frac{1}{2}(1+s+1)\right) \\
& =\frac{n m}{2}+\frac{n m}{4}-\frac{s}{2}+\frac{n m}{4}+1+\frac{s}{2} \\
& =n m+1 .
\end{aligned}
$$

For every odd value of $s \in\{1,2, \ldots, m\}$, the edge $v_{1,1} v_{2, \frac{n m}{2}}$ can be replaced with $v_{1, \frac{n m}{2}-s} v_{2,1+s}$ seeing that in this case

$$
\begin{aligned}
f\left(v_{1, \frac{n m}{2}-s}\right)+f\left(v_{2,1+s}\right) & =\frac{1}{2}\left(\frac{n m}{2}-s-1\right)+\left(\frac{3 n m}{4}+\frac{1+s}{2}\right) \\
& =\frac{n m}{4}-\frac{s}{2}-\frac{1}{2}+\frac{3 n m}{4}+\frac{1}{2}+\frac{s}{2} \\
& =n m
\end{aligned}
$$

If $u_{i, j} u_{i+1, j}$ is an edge of $G$ and $\phi\left(u_{i, j}\right) \phi\left(u_{i+1, j}\right)$ is not an edge of $C_{n m}$, then $\phi\left(u_{i, j}\right) \phi\left(u_{i+1, j}\right)$ must be used to replace the edge of $C_{n m}$ that has the same weight. Since this replacement is always possible (because $s$ exists in each case) without modifying neither the labels nor the weights, we conclude that the graph obtained after all the replacements are done is in fact $G$ with a strongly felicitous labeling, where the constant is $\lambda=\frac{n m}{2}$.

In Figure 9 we show the fifteen (non-isomorphic) members of $\mathscr{F}$ obtained with this construction for $n=4$ and $m=3$. Since the labeling for each of them is the same, we show the labeling of only one of these graphs.

\section{Conclusions}

We enumerated the harmoniously labeled graphs of size $n$ and order at most $n$. It is an open problem the enumeration of these labeled graphs when the order is $n+1$, case that includes all harmoniously labeled trees. We proved that for any odd cycle, attaching to some of its vertices any number of pendant vertices results in a harmonious graph. Is it possible to attach pendant paths instead of pendant vertices? We explored the corona of harmonious graphs and $K_{1}$, in this line, is it possible to replace $K_{1}$ by $m K_{1}$ ? We also proved that the union of a harmonious graph of order and size equal to $n$ with the start $K_{1, m}$ produces a harmonious graph. Could we replace the star by any other tree? Two large families of harmonious graphs were introduced: $n$-cell snake polyiamonds and unicyclic formed from the cycle $C_{4 t}$. Is it possible to build harmonious snakes using other shapes? And, instead of $C_{4 t}$, could we use $C_{2 t}$ with similar results? 

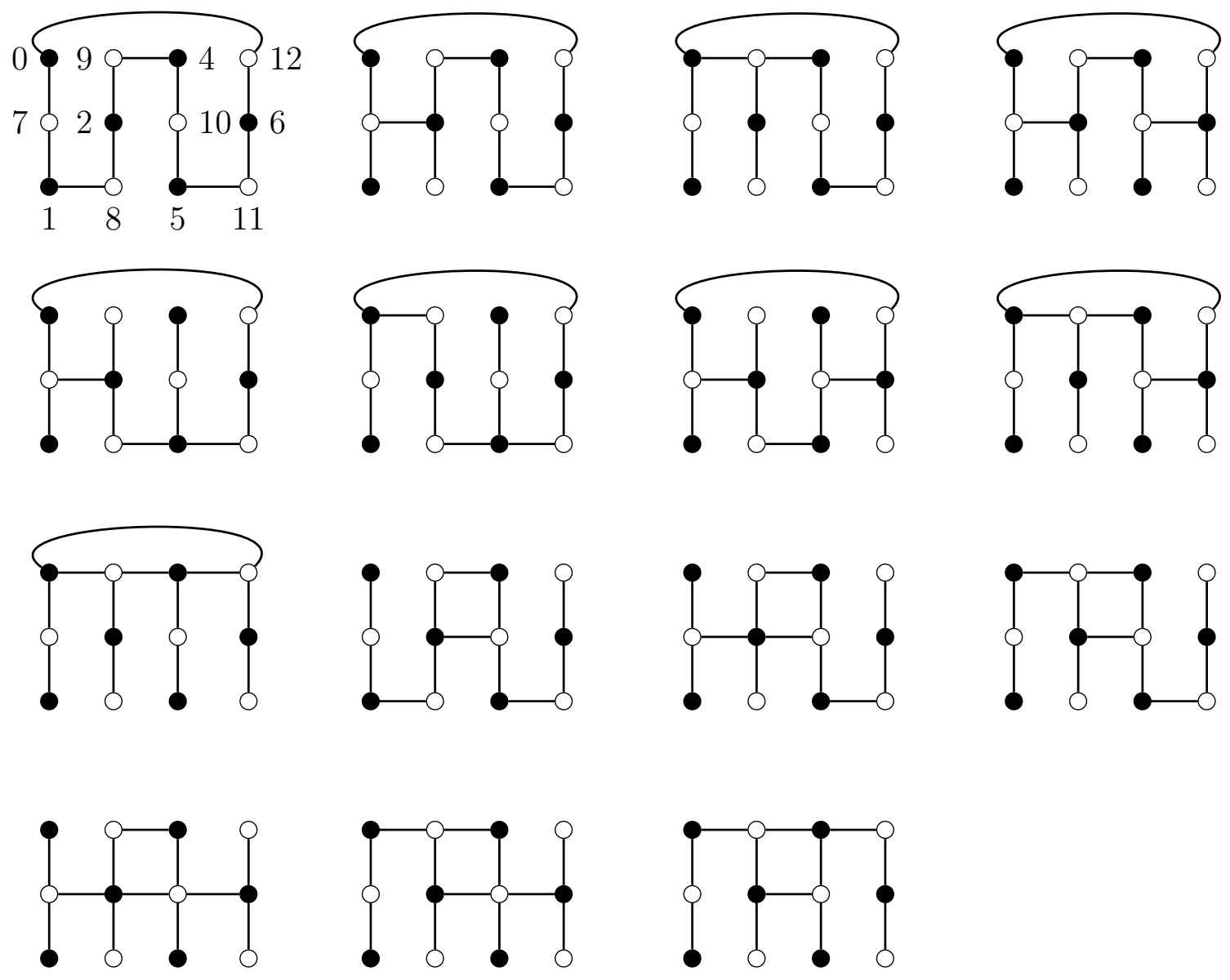

Figure 9. All non-isomorphic unicyclic graphs obtained with four copies of $P_{3}$

\section{Acknowledgement}

I would like to thank Sarah Minion for her help in the preparation of the manuscript and to the anonymous referee for her/his valuable comments, suggestions, and attentive reading of the manuscript.

\section{References}

[1] G. Agnarsson and R. Greenlaw, Graph Theory: Modeling, Applications, and Algorithms, Pearson, Upper Saddle River, 2006.

[2] M. Bača and M. Miller, Super Edge-Antimagic Graphs: A Wealth of Problems and Some Solutions, BrownWalker Press, Boca Raton, 2007

[3] C. Barrientos, Graceful graphs with pendant edges, Australas. J. Combin. 33 (2005), 99-107. 
[4] C. Barrientos and S. Minion, Snakes: from graceful to harmonious, Bull. Institute Combin. Appl., 79 (2017), 95-107.

[5] C. Barrientos, https://oeis.org/A005418, 2018.

[6] C. Barrientos, https://oeis.org/A329910, 2019.

[7] C. Barrientos, Special graceful labelings of irregular fences and lobsters, Univers. J. Math. Appl., 2 (1) (2019), 1-10.

[8] C. Barrientos and S. Minion, Counting and labeling grid related graphs, Electron. J. Graph Theory Appl., 7 (2) (2019), 349-363.

[9] R. Cattell, Graceful labellings of paths, Discrete Math., 307 (2007), 3161-3176.

[10] G. J. Chang, D. F. Hsu, and D. G. Rogers, Additive variations on a graceful theme: some results on harmonious and other related graphs, Cong. Numer, 32 (1981), 181-197.

[11] G. Chartrand and L. Lesniak, Graphs \& Digraphs 4th ed. CRC Press, Boca Raton, 2005.

[12] R. Figueroa-Centeno, R. Ichishima, and F. Muntaner-Batle, The place of super edge magic labelings among other classes of labelings, Discrete Math., 231 (2001), 153-168.

[13] R. Figueroa-Centeno, R. Ichishima, and F. Muntaner-Batle, Labeling the vertex amalgamation of graphs, Discuss. Math. Graph Theory, 23 (2003), 129-139.

[14] J. A. Gallian, A dynamic survey of graph labeling, Electron. J. Combin., (2019), \#DS6.

[15] T. Grace, On sequential labelings of graphs, J. Graph Theory, 7 (1983), 195-201.

[16] R. L. Graham and N. J. A. Sloane, On additive bases and harmonious graphs, SIAM J. Alg. Discrete Methods, 1 (1980), 382-404.

[17] A. Kotzig, On certain vertex valuations of finite graphs, Util. Math., 4 (1973), 67-73.

[18] B. Liu and X. Zhang, On a conjecture of harmonious graphs, Systems Sci. Math. Sci., 4 (1989), 325-328.

[19] S. C. López and F. A. Muntaner-Batle, Graceful, Harmonious and Magic Type Labelings: Relations and Techniques, Springer, Cham, 2017.

[20] J. Renuka, P. Balaganesan, and P. Selvaraju, On harmonious labeling, Internat. J. Advance in Math. Math. Sci., 1 (2) (2012), 65-70.

[21] A. Rosa, On certain valuations of the vertices of a graph, Theory of Graphs (Internat. Symposium, Rome, July 1966), Gordon and Breach, N. Y. and Dunod Paris (1967), 349-355.

[22] M. A. Seoud, A. E. I. Abdel Maqsoud, and J. Sheehan, Harmonious graphs, Util. Math., 47 (1995), 225-233. 
[23] G. Sethuraman and V. Murugan, Generating graceful unicyclic graphs from a given forest, to appear in AKCE Int. J. Graphs Combin.

[24] M. Truszcynski, Graceful unicyclic graphs, Demonstr. Math., 17 (1984), 377-387.

[25] W. D. Wallis, Magic Graphs, Birkhäuser, Boston, 2001.

[26] Y. Yang, W. Lu, and Q. Zeng, Harmonious graphs $C_{2 k} \cup C_{2 j+1}$, Util. Math., 62 (2002), 191198.

[27] M. Z. Youssef, Two general results on harmonious labelings, Ars Combin., 68 (2003), 225230. 